\title{
Biofortification - Associated Costs and Finding Alternatives?
}

Avik Ray [ORCID: 0000-0003-1662-7679] *

Center for studies in Ethnobiology, Biodiversity, and Sustainability (CEiBa), B.G. Road, Mokdumpur, Malda - 732103, West Bengal, India

*avikray@ceibatrust.org

\section{Acknowledgements}

I would like to thank Rajasri Ray for her suggestion that significantly improved the article. 


\begin{abstract}
Biofortification refers to the increase in the amount of essential vitamins or provitamins or minerals in crops to improve the nutritional status of the people, which is largely intended to alleviate the problem of micronutrient malnutrition. I argue that biofortification may not be an effective weapon to fight against the hidden hunger since it demonstrates limited capacity on nutritional enhancement and can negatively impact the socio-economic fabric of the society in many different ways. Finally, I suggest a couple of alternatives that might meet the challenge more efficiently than biofortified crops.
\end{abstract}

Keywords: Biofortification, Agro-biodiversity, HarvestPlus, Hidden Hunger, Orphan Crops, Wild edibles

Word (Text only) - 1938

\title{
Introduction
}


Nearly two billion people around the world suffer from low intakes of vitamins (e.g., Vitamin A) and key minerals (e.g., iron, zinc) (Horwitz et al. 1998). Micronutrient malnutrition or 'hidden hunger' now a global problem plaguing the human race. Hidden hunger may co-exist with the energy-rich staple crops (von Grebmer et al. 2014), as they are unable to erase micro-nutrient deficiency (Pinstrup-Andersen 2007). While dietary diversity has largely been mandated for healthy and nutritious food intake and to alleviate the burden of micronutrient malnutrition (FAO, WFP and IFAD. 2012), the potential of natural biodiversity in various capacities to enrich dietary diversity in general and to reduce micronutrient deficiency specifically is essentially ignored in food security policies and implementation. It is an issue of global concern when the world has gradually stepped in the realms of the biofortified crops that often portrayed as a panacea to eradicate hidden hunger, thus investing in the production of biofortified cereals, promoting, and spearheading the dissemination and molding the acceptance process at various levels (Birol and Bouis 2019).

In this article, I argue that biofortification may not be a better weapon to fight against hidden hunger. The implementation of biofortified crops could be deeply problematic as it hinges on the wrong assumption and may demonstrate their limited capacity to nutritional enhancement. Finally, I attempt to proffer a couple of alternatives that might meet the challenge more efficiently than do the biofortified crops.

\section{The game-changer - biofortification}

The technology of increasing the specific nutrient content of the crops through standard plant breeding or genetic engineering is termed as biological fortification or biofortification. It largely refers to the increase in the concentration of vitamins or provitamins or minerals in crops to improve nutritional status and has emerged to cure micronutrient deficiency or hidden hunger of the population of the developing world (Birol and Bouis 2019). Initially, three nutrients, vitamin A, 
iron, and zinc were at the forefront of concern. From then, about three hundred biofortified crops have been developed and released with the support from HarvestPlus which is a program of the Consultative Group on International Agricultural Research (CGIAR) and functions under the banner of CGIAR Research Program on Agriculture for Nutrition and Health (GRAIN 2019). These crops have been introduced into Asian, African, and South American countries and are now grown by ten million farmers and consumed by thirty-million people. The Golden Rice or GM rice high in provitamin A, provitamin A sweet potato, high iron beans or super banana or orange maize or biofortified pearl millet are some of the notable examples of biofortified crops. Many other crops are well underway to reach the market as well as the consumers' plates, e.g., GM cassava high in iron and zinc, GM sorghum high in provitamin A, zinc rice, pearl millet high in zinc and iron. Global research is currently ongoing to develop a range of crops biofortified with various nutrients, e.g., rice, wheat, sorghum, banana, lentil, potato, sweet potato, cassava, beans, and maize. The figure may soon skyrocket engulfing $90 \%$ of the crops and embracing the majority of the farming communities of the developing world (Yadav 2019).

\section{Problems of biofortification - handle with care}

A body of criticisms eclipse the proclaimed benefits of biofortified crops which can not be isolated as a simple attempt to alleviate hidden hunger, but it has serious intended or unintended consequences on the social, economic, and cultural fabric of the society where it has been introduced (Schnurr et al. 2018). Although their apparent cost-effectiveness and efficiency has also been vouched for their wider acceptance (De Steur et al. 2012; Meenakshi et al. 2010) there are several problems with the biofortified crops, their dissemination, and in reaching the final goal of nutritional enhancement.

First, cultivation of biofortified crops could seem to emerge as a vehicle for monoculture, while agricultural biodiversity, crop diversification, dietary diversity has largely been recommended for sustainable food systems (Ijaz M. et al. 2019; Frison et al 2019; Njeru, E.M., 2013 ), the premise has been thoroughly ignored 
in selective nutrient oriented advocacy of a handful of crops. Gradual embrace of biofortified crops would have a negative impact on indigenous agricultural biodiversity since the targeted regions of Asia, Africa, and South America are the centers of diversity or secondary centers of domestication of many crops including the major and minor cereals, vegetables, legumes. In the past, abolishment of hunger has been combated with the intensified production of three major cereals through the Green Revolution; it selectively relied only on a few nitrogen-responsive high yielding varieties with a narrow genetic base that became popular and widespread among the farmers (Dalrymple 1986). Therefore, we have witnessed an irreversible loss in crop genetic diversity following the Green Revolution (Pingali 2012). Impact was felt not only on staples, but also on other non-cereals due to cropping pattern change since wheat-rice based cropping systems became dominant, e.g., in India (Kataki, 2002). There are alarming examples from various parts of the globe that biofortified crops disregarded local diversity of cereals and vegetables despite their nutritional quality and resilience to local agro-climatic conditions, they also tend to disrupt local network to restore underutilized or orphan crops (GRAIN 2019). Tightly entangled to the issue of indigenous crop diversity is the case of seeds and food sovereignty that might be kept at stake with the wide-scale adoption of biofortified crops (Garcia-Casal et al. 2017). Furthermore, the creation of the desired crops with increased nutrients through the technology performs usual modification in addition to other existing agronomic manipulations pertaining to the crop yield, pest-resistance, or adaptation to environmental stresses. This potential can only be realized if other conditions for optimal agriculture are not limiting. Accumulation of minerals or other nutrients are often dependent on local environmental conditions in which the crop grows, so crops may or may not fully deliver the expected outcome of nutritional enhancement when grown under different agro-climatic conditions. It is even more imperative when nutrient depletion from soil affecting the crops has become a global trend now (Jones et al. 2013). On the same note, the bioavailability of nutrients is also a factor that finally dictates nutrient assimilation (Garcia-Casal et al. 2017).

Next, given the fact the dissemination of biofortified crops is a technocratic top-down approach that does not take into consideration of local cultural preference towards food that often is a complex blend of taste, 
color, texture, flavor or other ascertained tangible or intangible benefits of any crop, all of which determine how people accept and cherish their food; in other words, these local food systems are embedded within the culture. The case has been widely examined by Stone and Glover (2016) analyzing the acceptance of the Golden Rice in the Philippines. They concluded that the people of the Philippines have not accepted the Golden Rice with an open arm. Likewise, sweet potato is a major subsistence crop not a commodity in Tanzania, where it has been observed that women opted for biofortified sweet potato for making money by selling the same product and they have not considered the health aspect. Moreover, research on biofortified sweet potato cultivation also shows that women's labor and associated knowledge systems remained severely under- recognized (GRAIN 2019). So, the intrusion of these crops on social fabric can be damaging, as it disregards local resources, food systems, and peoples resilience. Additionally, it entirely relies on external funding, international relations, and access to social and economic assets; collectively, that is bound to offer only short-term benefits thus might not be a sustainable option (Rao 2018). Lastly, critics also fear that through this relatively simple process of biofortified crops adoption GM crops would gradually creep into the kitchen and can be circulated widely among the developing countries undermining indigenous diversity of bio-cultural resources. In the longer-term, it could slowly enable corporate control over our food.

\section{Finding better alternatives through the resurrection of deep-seated food culture}

Food is not simply a commodity, but it is endowed with gustatory, economic, cultural, and spiritual values (Counihan and Van Esterik 2012). So, it is a complex interaction of various attributes that finally decide the acceptance of food within a society. Food choice is deep-embedded in a culture and epitomizes the identity of an individual or a society or a group (Almerico 2014). However, lost in the midst of biofortification is the food culture that is a dynamic and long-nurtured tradition shaped up over generations - it may not be infiltrated through a top-down approach. For example, the standard diet of the south or 
south-east Asian people has always consisted of rice, leafy greens or salads along with a protein from various sources. Bangladeshi people consume rice with vegetables, fish, and lentils. Even the very poor will also have potato, leafy greens and lentils along with rice (Mazhar et al. 2007). In India, a bewilderingly diverse suite of leafy greens have been assimilated into regional cuisines for ages depending on the distribution, local and seasonal availability, taste (Pant 2019; Ray et al. 2020). So are the cases of other south, east, and south Asian countries, where leafy shoots occupy a major part of the diet and many a time are not cultivated but gathered at ease from the anthropogenic landscapes (Cruz-Garcia and Price, 2012). Locally procured leafy shoots are a rich source of vitamin A and contain many other minerals that can be effective against vitamin A deficiency. Similarly, wildly growing trees produce ripe or unripe fruits (Tamarindus indica, Zizyphus jujuba, Bassia latifolia, Avarhoea, Diospyros melanoxylon, Carissa canadus) which are often consumed raw or made into vegetables. A large diversity of species of flowers (Sesbania grandiflora, Moringa oleifera, Nelumbo nucifera, Nymphaea nouchali, Dregea volubilis) or leafy greens (Marsilea quadrifolia, Chenopodium album, Oxalis corniculata, Diplazium esculentum, Portulaca oleracea, etc) are devoured likewise. In short, our food culture is complex and diverse, imbibes a plethora of locally available resources apart from quintessential elements. To throw some light in this current debate on biofortification, therefore, we might find alternatives in traditional food systems that have been contingent on local resources and established over centuries. For combating various micronutrient malnutrition, we could rather evaluate scientifically, prioritize, and resurrect such a culture that is a key to the sustainable food system. In order to appreciate this, we could turn our face to resources other than staples or over-used crops, e.g., to wild edible biota, under-utilized or orphan crops that reserve untapped potential for achieving the goal of a balanced and nutritious diet. It is also crucial to consider the fact that these are already well-embedded in the local food systems, already ubiquitous in a variety of local cuisines depending on the season and relished widely (Mazhar et al. 2007; Pant 2019); it only deserves widespread sensitization, promotion, and proper policy intervention. 
Importantly, the integration of locally accessible resources into the diet has much larger implications in terms of environmental sustainability, when the world is burdened with food insecurity and climate change, and aiming to minimize agricultural footprints and slowly aligning with the priorities of sustainable food systems. Adoption and enhanced promotion of wild edible resources or resurrecting under-utilized or orphan crop calls for the attention of the policy-makers (Bharucha and Pretty 2010); since they offer several advantages, i.e., wide diversity, easy access to the local resource base, their availability, time-tested reliability, little or no management (Bharucha and Pretty 2010; Cruz-Garcia and Price, 2012). Summarily, their inclusion into the policy could emerge as a strategy not to tackle hidden hunger only but also to develop a sustainable food system.

\section{References:}


Almerico Gina M (2014): "Food and identity: food studies, cultural, and personal identity" Journal of International Business and Cultural Studies 8, $1 \mathrm{e} 7$.

Bharucha, Zareen and Jules, Pretty (2010) "The roles and values of wild foods in agricultural systems." Philosophical Transactions of the Royal Society B: Biological Sciences 365, no. 1554, pp 2913-2926.

Birol, Ekin and Bouis, Howarth E (2019) Leveraging biofortified crops and foods: R4D perspective. In Encyclopedia of Food Security and Sustainability Vol 2. Pp. 181-188. https://doi.org/10.1016/ B978-0-08100596-5.22450-5

Counihan, Carole, and Penny Van Esterik, eds. (2012) Food and culture: A reader. Routledge. Cruz-Garcia, Gisella S., and Lisa L. Price (2012) "Weeds as important vegetables for farmers." Acta Societatis Botanicorum Poloniae 81, no. 4, pp 397-403.

Dalrymple DG. 1986. Development and spread of high-yielding rice varieties in developing countries. Int. Rice Res. Inst.

De Steur, H., Gellynck, X., Blancquaert, D., Lambert, W., Van Der Straeten, D. and Qaim, M., 2012. Potential impact and cost-effectiveness of multi-biofortified rice in China. New Biotechnology, 29(3), pp.432-442.

FAO, WFP and IFAD. 2012. The State of Food Insecurity in the World 2012. Economic growth is necessary but not sufficient to accelerate reduction of hunger and malnutrition. Rome, FAO.

Frison, Emile A., Jeremy Cherfas, and Toby Hodgkin (2011) "Agricultural biodiversity is essential for a sustainable improvement in food and nutrition security." Sustainability 3, no. 1, pp 238-53.

Garcia-Casal, Maria Nieves, Juan Pablo Pena-Rosas, Boitshepo Giyose, Hans De Steur, and Dominique Van Der Straeten (2017) "Staple crops biofortified with increased vitamins and minerals: considerations for a public health strategy." Annals of the New York Academy of Sciences1390, no. 1, pp 3-13. 
von Grebmer, Klaus, Amy Saltzman, Ekin Birol, Doris Wiesman, Nilam Prasai, Sandra Yin, Yisehac Yohannes, Purnima Menon, Jennifer Thompson, and Andrea Sonntag (2014) Synopsis: 2014 Global Hunger Index: The Challenge of Hidden Hunger. Vol. 83. International Food Policy Research Institute 2014.

GRAIN 2019 Biofortified crops or biodiversity? The fight for genuine solutions to malnutrition is on. https://www.grain.org/e/6246 (last accessed on 4th March 2020)

Horwitz, Abraham, Eileen T. Kennedy, and Christopher P. Howson (1998) eds. Prevention of micronutrient deficiencies: tools for policymakers and public health workers. National Academies Press.

Ijaz, M., Nawaz, A., Ul-Allah, S., Rizwan, M.S., Ullah, A., Hussain, M., Sher, A. and Ahmad, S., 2019. Crop diversification and food security. In Agronomic crops (pp. 607-621). Springer, Singapore.

Jones, Davey L., Paul Cross, Paul JA Withers, Thomas H. DeLuca, David A. Robinson, Richard S. Quilliam, Ian M. Harris, David R. Chadwick, and Gareth Edwards-Jones (2013) "Nutrient stripping: the global disparity between food security and soil nutrient stocks." Journal of Applied Ecology 50, no. 4, pp 851-862.

Kataki, P. K. (2002). Shifts in cropping system and its effect on human nutrition: Case study from India. Journal of Crop Production, 6, 119-144.

Mazhar F, Buckles D, Satheesh PV and Akhter F (2007) Food sovereignty and uncultivated biodiversity in South Asia. New Delhi, India: Academic Foundation.

Meenakshi, J.V., Johnson, N.L., Manyong, V.M., DeGroote, H., Javelosa, J., Yanggen, D.R., Naher, F., Gonzalez, C., Garcia, J. and Meng, E., 2010. How cost-effective is biofortification in combating micronutrient malnutrition? An ex ante assessment. World Development, 38(1), pp.64-75. 
Njeru, E.M., 2013. Crop diversification: a potential strategy to mitigate food insecurity by smallholders in sub-Saharan Africa. Journal of Agriculture, Food Systems, and Community Development, 3(4), pp.63-69.

Pant Pushpesh (2017) “Green forever”. In: first food - culture of taste, Narain Sunita, Mishra Aditya, Talwar Priya, Mahapatra Richard (eds). Center for Science and Environment, New Delhi Pingali, Prabhu L (2012) "Green revolution: impacts, limits, and the path ahead." Proceedings of the National Academy of Sciences 109, no. 31, pp. $12302-12308$.

Pinstrup-Andersen, P (2007) "Agricultural research and policy for better health and nutrition in developing countries: a food systems approach." Agricultural Economics 37, pp 187-198.

Rao, Sheila. "Sweet Success? Interrogating Nutritionism in Biofortified Sweet Potato Promotion in Mwasonga, Tanzania." (Doctoral dissertation, Carleton University).

Ray A, Ray R and Sreevidya EA (2020) How Many Wild Edible Plants Do We Eat-Their Diversity, Use, and Implications for Sustainable Food System: An Exploratory Analysis in India. Frontiers in Sustainable Food System 4:56. doi: 10.3389/fsufs.2020.00056

Schnurr, Matthew A., Lincoln Addison, and Sarah Mujabi-Mujuzi (2020) "Limits to biofortification: Farmer perspectives on a vitamin a enriched banana in Uganda." The Journal of Peasant Studies 47, no. 2 , pp 326-345.

Stone, Glenn Davis, and Dominic Glover (2017) "Disembedding grain: Golden Rice, the Green Revolution, and heirloom seeds in the Philippines." Agriculture and Human Values 34, no. 1, pp 87-102.

Swagata Yadavar (2019) "Fighting hidden hunger: 'Our mission is 90\% of crops must be biofortified"”, IndiaSpend, 17 February 2019, https://www.indiaspend.com/fighting-hidden-hunger- our-mission- is-90of-crops-must-be-biofortified/is[ipe] 
